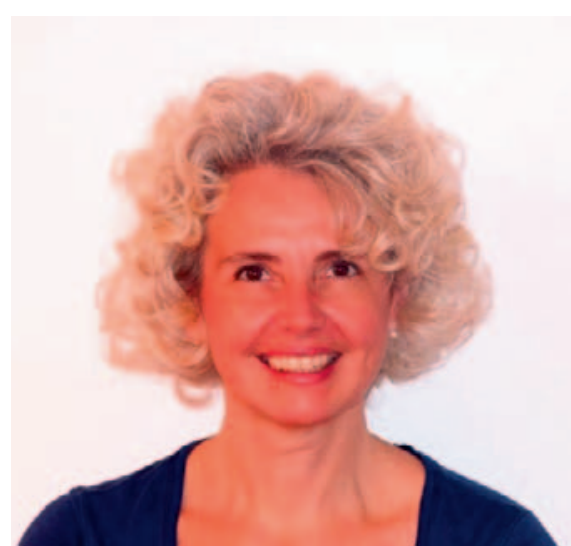

Sehr geehrte Frau

Dr. Zahradnicek, bitte geben

Sie uns einen kurzen

Überblick, mit welchen

Behandlungsschwerpunkten

Sie sich innerhalb der

Komplementärmedizin vor

allem befassen.

Komplementärmedizin bedeutet für mich eine Vielfalt von Betrachtungs- und Behandlungsweisen. Eine besondere Rolle spielen bei mir Akupunktur, Phytotherapie und Hypnose, welche eher zu den psychotherapeutischen Verfahren gehört.

Mit diesen unterschiedlichen Verfahren beziehe ich unterschiedliche Heilungsebenen in den Gesundungsprozess mit ein:

(1) Körperliche Ebene.

(2) Energetische Ebene.

(3) Psychische Ebene, sowohl die des Bewussten als auch die des Unbewussten.

Für mich ist vor allem die Phytotherapie ein zentraler Bestandteil komplementärmedizinischer bzw. naturheilkundlicher Vorgehensweisen. Sie bietet mir unterschiedliche therapeutische Ebenen des Arbeitens an. Neben der körperlichen Seite von Erkrankungen ermöglichen mir die Heilpflanzen noch andere therapeutische Ansätze: Da sind

\title{
Vielschichtige komplementärmedizinische Betrachtungsweisen
}

Frau Dr. Simona Zahradnicek ist heute im Medicum Wesemlin in Luzern sowie in der komplementärmedizinischen Beratung und Mitbehandlung onkologischer Patienten im OnkoZentrum Zürich und im Onkozentrum Hirslanden Zürich tätig. Zuvor war sie als Fachärztin am Institut für Naturheilkunde des Universitätsspitals Zürich aktiv.

auf der einen Seite das Wesen der Pflanze und auf der anderen Seite die kulturellen Aspekte und die eigenen, persönlichen Erfahrungen. Diese Gesichtspunkte geben der Pflanze im Vergleich zu den wissenschaftlich anerkannten Aspekten noch eine zusätzliche personenzentrierte Bedeutung und Wirkung. Ich kann einerseits die verschiedenen Ebenen patienten- und krankheitsorientiert miteinander verbinden und andererseits nur einzelne Gesichtspunkte herausgreifen. Damit meine ich, dass die Pflanzen ihren Heilcharakter und ihre Indikationsgebiete nicht nur auf der körperlichen Ebene haben. Sie können bei der Gesundung auch mit ihrem Wesen seelisch auf einen Patienten einwirken. Dies mache ich mir häufig bei psychosomatischen oder psychischen Erkrankungen zunutze.

\section{Sie haben im Rahmen ihrer Tätigkeit Aufenthalte in Grossbritannien, Italien, Deutschland und Australien absolviert. Welchen Einfluss haben Ihre Erfahrungen aus anderen Ländern auf Ihre ärztliche Vorgehensweise?}

Die Auslandsaufenthalte haben meinen Blick geweitet. Kulturelle Tra- ditionen und Gesichtspunkte spielen in der Therapie eine grosse Rolle. Es fällt mir viel leichter, den Patienten zu verstehen und seine Gedankengänge nachzuvollziehen. Darüber hinaus haben mir die Aufenthalte in Grossbritannien und Australien gezeigt, wie wichtig der partnerschaftliche Umgang unter Kollegen ist.

\section{Sie sind als selbstständige \\ Fachärztin derzeit im \\ Medicum Wesemlin \\ in Luzern sowie in der komplementärmedizinischen Beratung und Mitbehandlung onkologischer Patienten im OnkoZentrum Zürich, Klinik im Park, und im Onkozentrum Hirslanden Zürich tätig. Was ist das Leitbild dahinter?}

Das Medicum Wesemlin ist eine transdisziplinäre und interdisziplinäre medizinisch-therapeutische Einrichtung für ambulante Medizin, welche im Frühjahr 2015 in den Räumlichkeiten eines 1584 gegründeten Kapuzinerklosters in der Stadt Luzern realisiert wurde. In konzeptioneller Hinsicht werden schulmedizinische und komplementärmedizinische Vorgehensweisen patienten- und krank- 
heitszentriert in einem Team von Allgemeinmedizinern, Spezialisten und nichtärztlichen Therapeuten verschiedener Fachdisziplinen umgesetzt. Was ich persönlich als sehr schön empfinde, ist die Nähe zu den Kapuzinerbrüdern und ihre Einbindung um das persönliche Wohl des Patienten.

Was die Onkozentren betrifft:

Für tumorkranke Menschen ist es ausserordentlich wichtig, dass auch komplementärmedizinische Betrachtungsweisen, soweit sinnvoll, in die Therapien einbezogen werden. Genau dies versuche ich in meiner konsiliarischen Tätigkeit in beiden onkologischen Praxen umzusetzen. Und dies ist auch der Wunsch der Onkologen, mit denen ich eng zusammenarbeite. Die Sprechstunde ist ebenso offen für Patienten, die ihre onkologische Behandlung bei anderen Institutionen durchführen, oder für Angehörige, die eine Beratung wünschen.

\section{Kürzlich haben Sie an einem internationalen Kongress zur Pflanzenheilkunde einen Beitrag zur Pleiotropie der verschiedenen Minzen vorgestellt. Was ist Ihrer Meinung nach an den Minzen ganz besonders? Bei welchen Beschwerden eignen sich diese ganz speziell?}

Die Minze ist für mich paradigmatisch ein Beispiel für die Vielfalt und Mannigfaltigkeit der therapeutischen Möglichkeiten, die in Arznei- und Heilpflanzen nahezu regelhaft enthalten sind. Sie bietet die äusserliche sowie die innerliche Anwendung und über die ätherischen Öle zusätzlich ein erweitertes Indikationsspektrum.

Neben der belegbaren pharmakologischen Pleiotropie spielen bei den Wirkungen auf den gesamten Organismus individuell interpretierte olfaktorische Sensationen eine nicht zu unterschätzende Rolle. So können Phytotherapeutika allgemein, und dies gilt nicht nur für Minzen, auch über
Sinneskanäle therapeutische individuelle Wirkungen erzielen oder auch vermindern. Mit Düften wird der phylogenetisch älteste Sinn angesprochen. Geruchseindrücke sind häufig mit anderen Erfahrungen und den damit assoziierten körperlichen und seelischen Reaktionen gekoppelt bzw. koppelbar und können diese bei Exposition auslösen. Mir war wichtig, dies aufzuzeigen.

Die verschiedenen Minzen finden ethnomedizinisch, insbesondere auch in europäischen Traditionen, ein breites Anwendungsspektrum und können vergleichbar verwendet werden. Anhand des Vergleichs der unterschiedlichen Medizinkonzepte (westliche Phytotherapie, Traditionelle Chinesische Medizin (TCM) und arabische Medizin/Unami-Medizin) und der damit einhergehenden Verwendung verschiedener Minzen wird aufgezeigt, wie die Minzen bei identischer Indikation dieselbe Wirkung zeigen, die Darreichungsform und -art sowie der ihnen zugeordnete Wirkmechanismus je nach Medizinsystem jedoch differieren können.

Einen Teil der traditionellen Anwendungsgebiete finden wir in allen drei genannten Medizinsystemen:

- Innerlich: Meteorismus, funktionelle Magen-Darm-Galle-Beschwerden, Katarrhe der äusseren Luftwege.

- Äusserlich: Myalgien und Neuralgien.

Der Beforschungsstand der einzelnen Minzen ist derzeit uneinheitlich. Die meisten berichteten Anwendungsgebiete konnten mittlerweile wissenschaftlich bestätigt werden, und zum Teil konnten die Indikationen durch die moderne Forschung sogar erweitert werden.

Zur Fülle der pharmakologischen Forschungsergebnisse gehören folgende Wirkungen: spasmolytisch, choleretisch, karminativ, Förderung der Magensaftsekretion, appetitanregend, adstringierend, antiviral, antibakteriell, antimykotisch, antinozeptiv, antientzündlich, antioxidativ, diuretisch, sekretolytisch, kühlend, lokal anästhesierend, hyperämisierend, schleimhautabschwellend.
Zur Erweiterung aufgrund der aktuellen Forschung gehören: kognitionsverbessernd, radioprotektiv, Hirsutismus abschwächend, apoptotisch, fakultativ sedierend.

Der Forschungsschwerpunkt liegt derzeit bei der Pfefferminze.

\section{Wie sind Sie selbst ursprünglich mit der Komplementärmedizin in Berührung gekommen? Was fasziniert Sie daran am meisten?}

In einer fantasievollen qualitätsorientierten Komplementärmedizin, vor allem aber in der Phytotherapie, lassen sich die Denk- und Erfahrungswelten der Patienten aufgreifen und mit den Vorstellungen der modernen Medizin verbinden. Zugleich ist es möglich, traditionell verankert zu bleiben.

Über längere Zeit habe ich mich mit der TCM beschäftigt. Während des Studiums der chinesischen Heilkräuter fingen mehr die westlichen, d.h. die europäischen, Pflanzen an, mich zu interessieren, da sie zu unseren kulturellen Traditionen gehören und ich einen grösseren Bezug zu ihnen verspürte.

Seit einigen Jahren bin ich Mitglied der Schweizerischen Medizinischen Gesellschaft für Phytotherapie. Dort habe ich im Rahmen der Phytotherapieausbildung meine Kenntnisse deutlich erweitert. Noch während meiner Zeit in Deutschland habe ich eine Reihe von Kursen zur ärztlichen Ausbildung in Naturheilverfahren absolviert. Mein grösstes Wissen habe ich in den beiden Jahren meiner Tätigkeit als Fachärztin am ehemaligen Institut für Naturheilkunde des Universitätsspitals Zürich durch den damaligen Institutsdirektor Prof. Dr. med. Reinhard Saller erworben.

\section{Liebe Frau Dr. Zahradnicek, haben Sie herzlichen Dank für das Interview!}

Interview: Alexander Eitner 